\title{
Detecting EGFR mutations (L858R, T790M) using allele specific multiplex sequencing: A comparison with Pyrosequencing and TruSeq
}

\author{
Dilanthi Vinayagamoorthy ${ }^{1}$, Jennifer Walsh ${ }^{1}$, Kierra Gipson ${ }^{1}$, Fei Ye ${ }^{3}$, Minghao Zhong ${ }^{3}$, Qin Dahui ${ }^{2}$, Thuraiayah \\ Vinayagamoorthy*1 \\ ${ }^{1}$ Select MultiGEN Diagnostics, Greensboro, NC, United States \\ ${ }^{2}$ Molecular Diagnostic Lab, Moffitt Cancer Center, FL, United States \\ ${ }^{3}$ Westchester Medical Center, Valhalla, NY, United States
}

Received: December 23, 2019

Accepted: February 16, 2020

Online Published: March 6, 2020

DOI: $10.5430 /$ jst.v10n1p19

URL: https://doi.org/10.5430/jst.v10n1p19

\begin{abstract}
We are presenting an evaluation of Allele Specific Multiplex Sequencing (ASMS) to detect two EGFR somatic mutations (L858R, T790M). Late stage lung cancer samples were tested for both EGFR mutations and were compared to either pyrosequencing or TruSeq. The analytical lower limit of detection (LLOD) for the ASMS-L858R assay was found to be 36 copies, and 72 copies for the ASMS-T790M assay. The forty-one FFPE samples that were tested for T790M showed $100 \%$ concordance with the respective comparative method. The forty-five FFPE samples tested previously by Truseq for L858R showed 100\% concordance with ASMS. Out of the twenty L858R samples previously tested by pyrosequencing, there was 95\% concordance with ASMS. Additionally, twenty-one normal blood samples were tested by ASMS were found to be negative for L858R and T790M. In conclusion, the detection of L858R and T790M by ASMS are in acceptable concordance with both pyrosequencing and TruSeq in detecting EGFR mutations from late stage lung cancer. Further, ASMS was able to detect EGFR (L858R) with 10 picograms (3 copies gDNA) of FFPE extracted DNA, and hence could be used to detect mutations from samples carrying low copy numbers.
\end{abstract}

Key Words: ASMS, EGFR, L858R, T790M, Lung cancer

\section{INTRODUCTION}

Targeted chemotherapy is one of the therapeutic modalities used in the management of late stage cancers. Late stage non-small cell lung cancer (NSCLC) is treated with specific targeted chemotherapy subject to detecting specific Epidermal Growth Factor receptor (EGFR) somatic mutations. ${ }^{[1,2]}$ Two of the 'actionable' EGFR mutations are L858R and T790M, both of which are associated with lung cancer. ${ }^{[3,4]}$ These markers are routinely tested using DNA extracted from FFPE samples, and use of specific targeted chemotherapies has shown clinical utility. Having documented clinical utility in stage IV cancers, studies are underway to explore use of targeted chemotherapies in early stage cancers (< stage IIIa, IIIb, II). A previous report shows that detection of Braf p. V600E/K mutations was significantly higher using ASMS compared to two of the presently used technologies. Further, early stage cancers tend to have less cancer burden than stage IV cancers, and hence may carry a fewer number of copies of targeted chemotherapy specific somatic mutations. Therefore, detection of actionable mutations in early stage

${ }^{*}$ Correspondence: Thuraiayah Vinayagamoorthy; Email: moorthy@multigen-diagnostics.com; Address: Select MultiGEN Diagnostics, Greensboro, NC 27405, United States. 
cancers may require assays that are more sensitive than what is currently available.

Prior to engagement in detecting EGFR L858R and T790M in early stage cancers, the assay requires clinical validation. This study presents results of a clinical validation of detecting EGFR L858R and T790M mutations using Allele specific Multiplex Sequencing (ASMS) technology, and comparison to pyrosequencing and TruSeq. ${ }^{[6-9]}$ Further, clinical lower limit of detection was determined using serially diluted positive EGFR L858R and T790M biopsy samples.

\section{MATERIALS AND METHODS}

\subsection{Samples}

The study included DNA controls (Horizon Discoveries UK), twenty-one blood samples from asymptomatic normal population and one hundred and six de-identified late stage lung cancer DNA extracts from FFPE samples.

\subsection{Method}

DNeasy Blood (Qiagen, USA) was used according to manufacturer's instructions to obtain DNA from $1 \mathrm{ml}$ of blood. Ten ul of the DNA extract was used per PCR reaction. Amplification and cycle sequencing were performed separately for T790M and L858R, according to manufacturer's instructions (MultiGEN Diagnostics Inc, USA).

Table 1. ASMS EGFR (T790M) Lower limit of detection

\begin{tabular}{|c|c|c|}
\hline Serial dilution & $\begin{array}{l}\text { Copies of EGFR- } \\
\text { T790M/Rxn }\end{array}$ & Results \\
\hline Stock solution & $5 n g / u l$ & $\mathrm{~N} / \mathrm{T}$ \\
\hline $1: 10$ & 7,250 & T790M Positive \\
\hline $1: 100$ & 725 & T790M Positive \\
\hline $1: 1000$ & 73 & T790M Positive \\
\hline $1: 10000$ & 7 & T790M Negative \\
\hline $1: 100000$ & $<1.0$ & T790M Negative \\
\hline Negative & 0 & Negative \\
\hline
\end{tabular}

Table 2. ASMS EGFR ( L858R) Lower limit of detection

\begin{tabular}{lll}
\hline Serial dilution & $\begin{array}{l}\text { Copies of } \\
\text {-L858R/Rxn }\end{array}$ & Results \\
\hline Stock solution & $5 \mathrm{ng} / \mathrm{ul}$ & \\
Original & 730 & L858R Positive \\
$1: 2$ & 365 & L858R Positive \\
$1: 4$ & 182 & L858R Positive \\
$1: 10$ & 73 & L858R Positive \\
$1: 20$ & 36 & L858R Positive \\
$1: 40$ & 18 & L858R Negative \\
Negative & 0 & Negative \\
\hline
\end{tabular}

\section{RESUlts AND Discussion}

\subsection{Sensitivity}

Lower limit of detection of L858R and T790M was determined using hgDNA from human cell line. (Horizon Discoveries Cambridge, UK.). Using serial dilutions of the human cell line extracted DNA stock solution (5ug/ul), the lower limit of detection was 36 copies for L858R and 73 copies for T790M (see Tables 1, 2).

Table 3. Primer Specificity of ASMS for detection of EGFR L858R

\begin{tabular}{lll}
\hline Template & Sequencing Primer & Electropherogram \\
\hline Mutant & Mutant-L858R & Mutant signal \\
Mutant & Wild-L858L & Negative \\
Wild & Wild-L858L & Wild type signal \\
Wild & Mutant-L858R & Negative \\
\multirow{2}{*}{ Wild /Mutant } & Mutant-L858R & Mutant \& Wild type \\
& \&Wild-L858L & signal \\
\hline
\end{tabular}

Table 4. Primer Specificity of ASMS for detection of EGFR T790M

\begin{tabular}{lll}
\hline Template & Squencing primer & Electropherogram \\
\hline Mutant & Mutant-T790M & Mutant signal \\
Mutant & Wild-T790T & Negative \\
Wild & Wild-T790T & Wild type signal \\
Wild & Mutant-T790M & Negative \\
\multirow{2}{*}{ Wild/Mutant } & Mutant-T790M & Mutant \& Wild type \\
& \& Wild-T790T & signal \\
\hline
\end{tabular}

\subsection{Specificity}

\subsubsection{L858R and T790M}

Using plasmid controls (Integrated DNA Technologies, USA), specificities of the sequencing primers were determined. L858R and T790M were tested for specificity separately. The mutant sequencing primer and the wild type sequencing primer with their respective control plasmid templates generated expected nucleotide sequences (see Tables $3,4)$. The mutant sequencing primer with wild type template, and wild type sequencing primer with mutant plasmid template did not generate a nucleotide sequence. When both sequencing primers were added into a reaction with wild type controls, only wild type sequence was generated (see Figure 1,2). When both sequencing primers were added into a reaction with mutant plasmid and wild type controls, this generated two sequences; one from the mutant and the other from the wild type at the respective locus (see Figure $3,4)$. In addition, all twenty-one normal blood samples that were tested for EGFR L858R and T790M were negative for mutation (see Table 5). 
Table 5. Comparison of ASMS ( EGFR T790M and L858R) with Pyrosequencing and TruSeq

\begin{tabular}{|c|c|c|c|c|c|c|}
\hline & \multicolumn{2}{|l|}{ Samples } & \multicolumn{4}{|l|}{ Results } \\
\hline \multirow[t]{3}{*}{ Marker } & Type & Number of samples tested & Allele Status & ASMS & Pyrosequencing & TruSeq \\
\hline & & & T790M & 0 & NT & NT \\
\hline & Normal blood & 21 & Negative & 21 & NT & NT \\
\hline \multirow{4}{*}{ T790M } & \multirow{4}{*}{ FFPE } & \multirow{2}{*}{3} & T790M & 0 & 0 & - \\
\hline & & & Negative & 3 & 3 & _ \\
\hline & & \multirow{2}{*}{38} & T790M & 7 & - & 7 \\
\hline & & & Negative & 31 & - & 31 \\
\hline \multirow{6}{*}{ L858R } & \multirow{2}{*}{ Normal blood } & \multirow{2}{*}{21} & L858R & 0 & NT & NT \\
\hline & & & Negative & 21 & NT & NT \\
\hline & \multirow{4}{*}{ FFPE } & \multirow{2}{*}{20} & L858R & 4 & 3 & - \\
\hline & & & Negative & 16 & 17 & _- \\
\hline & & \multirow{2}{*}{45} & L858R & 3 & - & 3 \\
\hline & & & Negative & 42 & - & 42 \\
\hline Total & & 148 & & & & \\
\hline
\end{tabular}

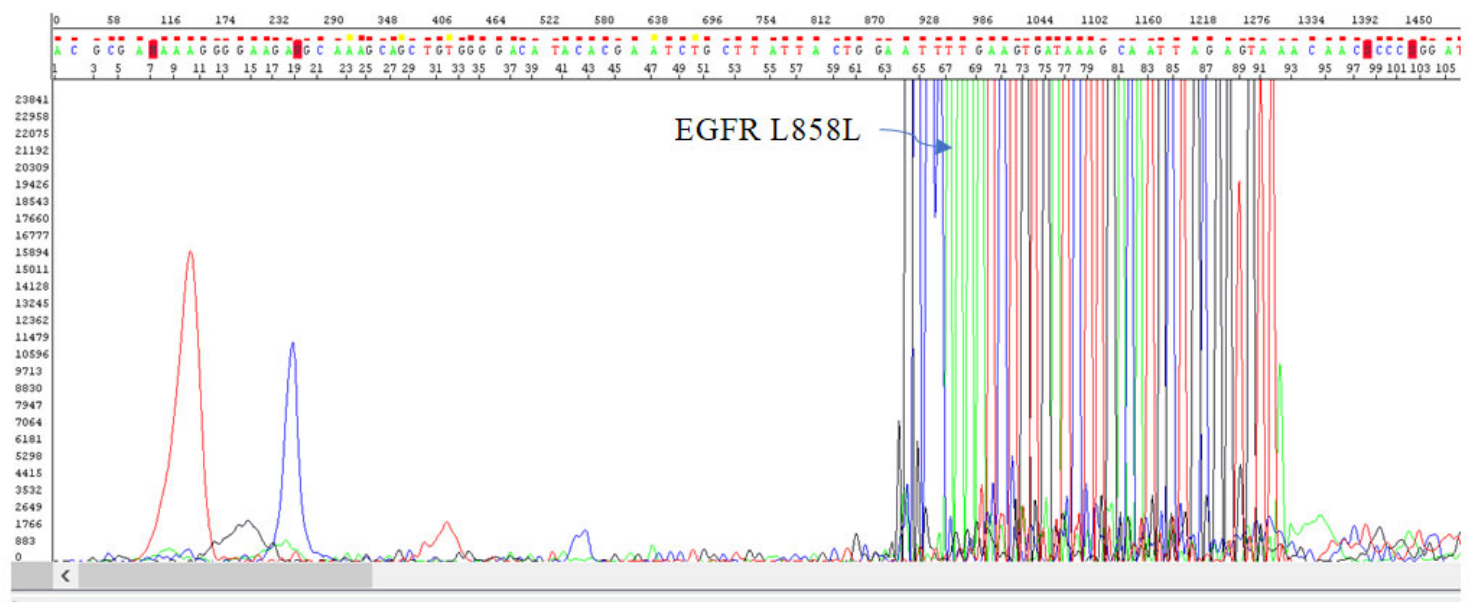

Figure 1. Electropherogram of a L858R negative FFPE sample

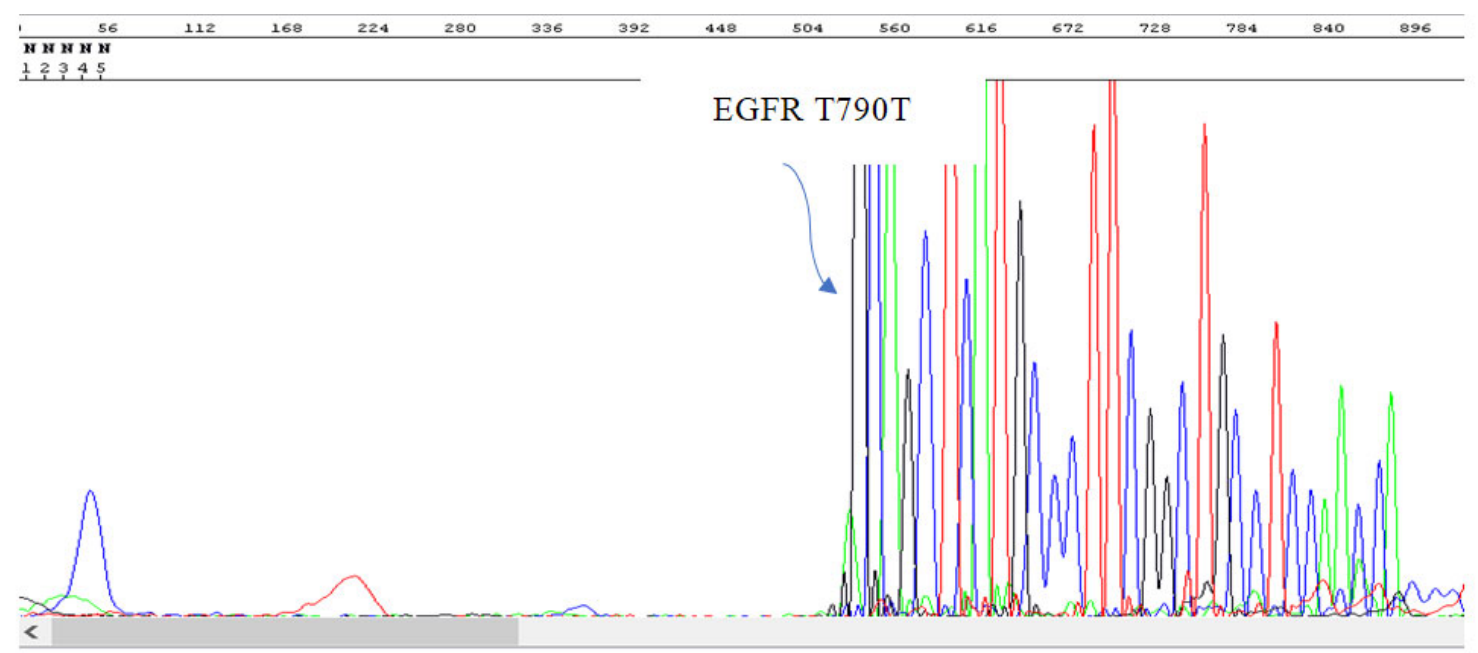

Figure 2. Electropherogram of a T790M negative FFPE sample 
Table 6. Serial dilution of FFPE sample EGFR L858R

\begin{tabular}{|c|c|c|c|c|c|c|c|}
\hline Amount of DNA/sample & $\begin{array}{l}\text { No. of mutant } \\
\text { copies/sample }\end{array}$ & Sample 1 & Sample 2 & Sample 3 & Sample 4 & Sample 5 & Sample 6 \\
\hline lng & 300 & $\mathrm{M} / \mathrm{W}$ & $\mathrm{M} / \mathrm{W}$ & $\mathrm{M} / \mathrm{W}$ & $\mathrm{M} / \mathrm{W}$ & $\mathrm{M} / \mathrm{W}$ & $\mathrm{M} / \mathrm{W}$ \\
\hline $0.1 \mathrm{ng}$ & 30 & $\mathrm{M} / \mathrm{W}$ & $\mathrm{M} / \mathrm{W}$ & $\mathrm{M} / \mathrm{W}$ & $\mathrm{M} / \mathrm{W}$ & $\mathrm{M} / \mathrm{W}$ & $\mathrm{M} / \mathrm{W}$ \\
\hline $0.01 \mathrm{ng}$ & 3 & Neg & Neg & Neg & $\mathrm{M} / \mathrm{W}$ & Neg & $\mathrm{M} / \mathrm{W}$ \\
\hline $0.001 \mathrm{ng}$ & 0 & $\mathrm{Neg}$ & Neg & Neg & Neg & $\mathrm{Neg}$ & $\mathrm{Neg}$ \\
\hline $0.0001 \mathrm{ng}$ & 0 & $\mathrm{Neg}$ & $\mathrm{Neg}$ & $\mathrm{Neg}$ & $\mathrm{Neg}$ & $\mathrm{Neg}$ & $\mathrm{Neg}$ \\
\hline $0.00001 \mathrm{ng}$ & 0 & $\mathrm{Neg}$ & $\mathrm{Neg}$ & $\mathrm{Neg}$ & $\mathrm{Neg}$ & NT & $\mathrm{Neg}$ \\
\hline $0.000001 \mathrm{ng}$ & & $\mathrm{Neg}$ & NT & NT & NT & NT & NT \\
\hline Neg & & $\mathrm{Neg}$ & Neg & Neg & $\mathrm{Neg}$ & Neg & $\mathrm{Neg}$ \\
\hline
\end{tabular}

Table 7. Serial dilution of FFPE sample EGFR T790M

\begin{tabular}{lllllllll}
\hline Amount of DNA/sample & $\begin{array}{l}\text { No. of mutant } \\
\text { copies/sample }\end{array}$ & Sample 1 & Sample 2 & Sample 3 & Sample 4 & Sample 5 & Sample 6 \\
\hline $1 \mathrm{ng}$ & 300 & $\mathrm{~W}$ & $\mathrm{~W}$ & $\mathrm{~W}$ & $\mathrm{~W}$ & $\mathrm{~W}$ & $\mathrm{~W}$ \\
$0.1 \mathrm{ng}$ & 30 & $\mathrm{~W}$ & $\mathrm{Neg}$ & $\mathrm{W}$ & $\mathrm{Neg}$ & $\mathrm{W}$ & $\mathrm{W}$ \\
$0.01 \mathrm{ng}$ & 3 & $\mathrm{Neg}$ & $\mathrm{Neg}$ & $\mathrm{Neg}$ & $\mathrm{Neg}$ & $\mathrm{W}$ & $\mathrm{W}$ \\
$0.001 \mathrm{ng}$ & 0 & $\mathrm{Neg}$ & $\mathrm{Neg}$ & $\mathrm{Neg}$ & $\mathrm{Neg}$ & $\mathrm{Neg}$ & $\mathrm{Neg}$ \\
$0.0001 \mathrm{ng}$ & 0 & $\mathrm{Neg}$ & $\mathrm{Neg}$ & $\mathrm{Neg}$ & $\mathrm{Neg}$ & $\mathrm{Neg}$ & $\mathrm{Neg}$ \\
$0.00001 \mathrm{ng}$ & 0 & $\mathrm{Neg}$ & $\mathrm{Neg}$ & $\mathrm{Neg}$ & $\mathrm{Neg}$ & $\mathrm{Neg}$ & $\mathrm{Neg}$ \\
$0.000001 \mathrm{ng}$ & & $\mathrm{NT}$ & $\mathrm{NT}$ & $\mathrm{NT}$ & $\mathrm{NT}$ & $\mathrm{NT}$ & $\mathrm{NT}$ \\
\hline
\end{tabular}

Note. $\mathrm{M}=$ Mutant allele; $\mathrm{W}=$ Wild-type allele; $\mathrm{NT}=$ Not Tested; Neg= Negative $* 1$ ng of genomic DNA= approximately 300 copies of mutant allele, given $100 \%$ tumor content

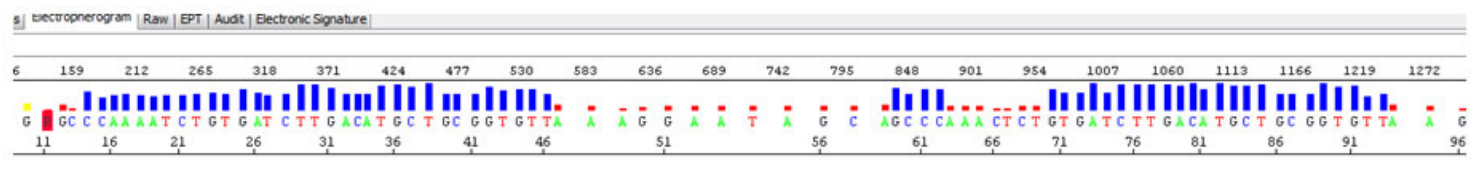

EGFR L858R

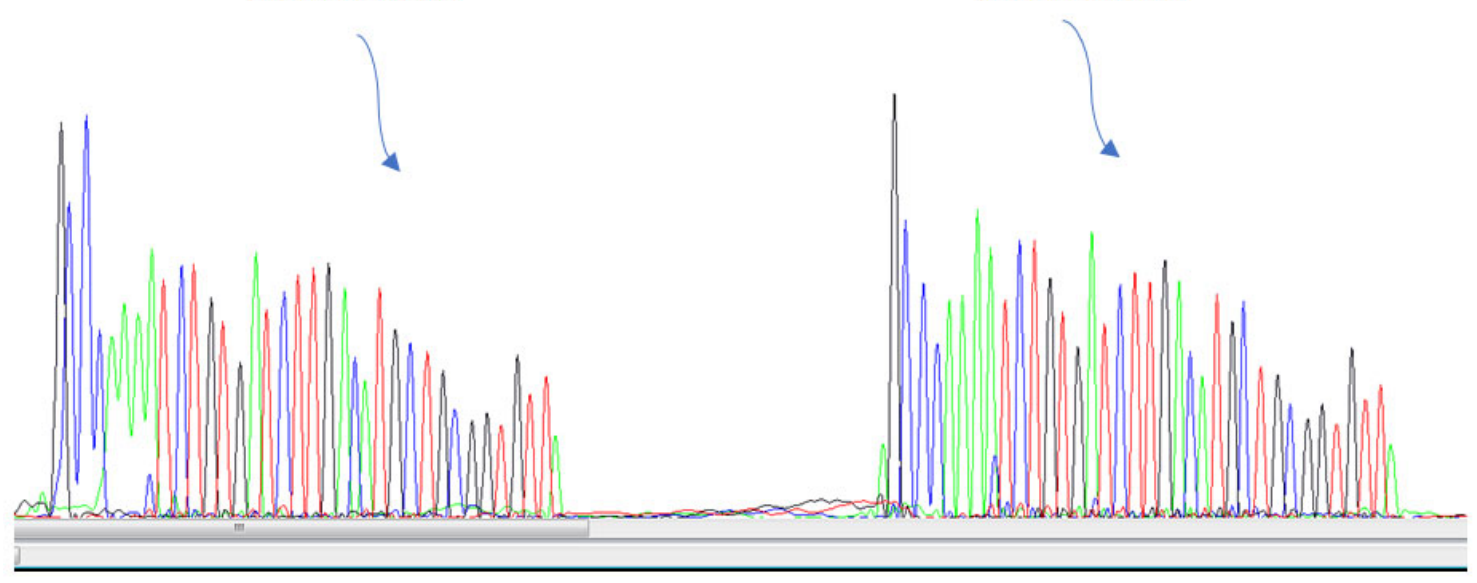

Figure 3. Electropherogram of a L858R positive FFPE sample 


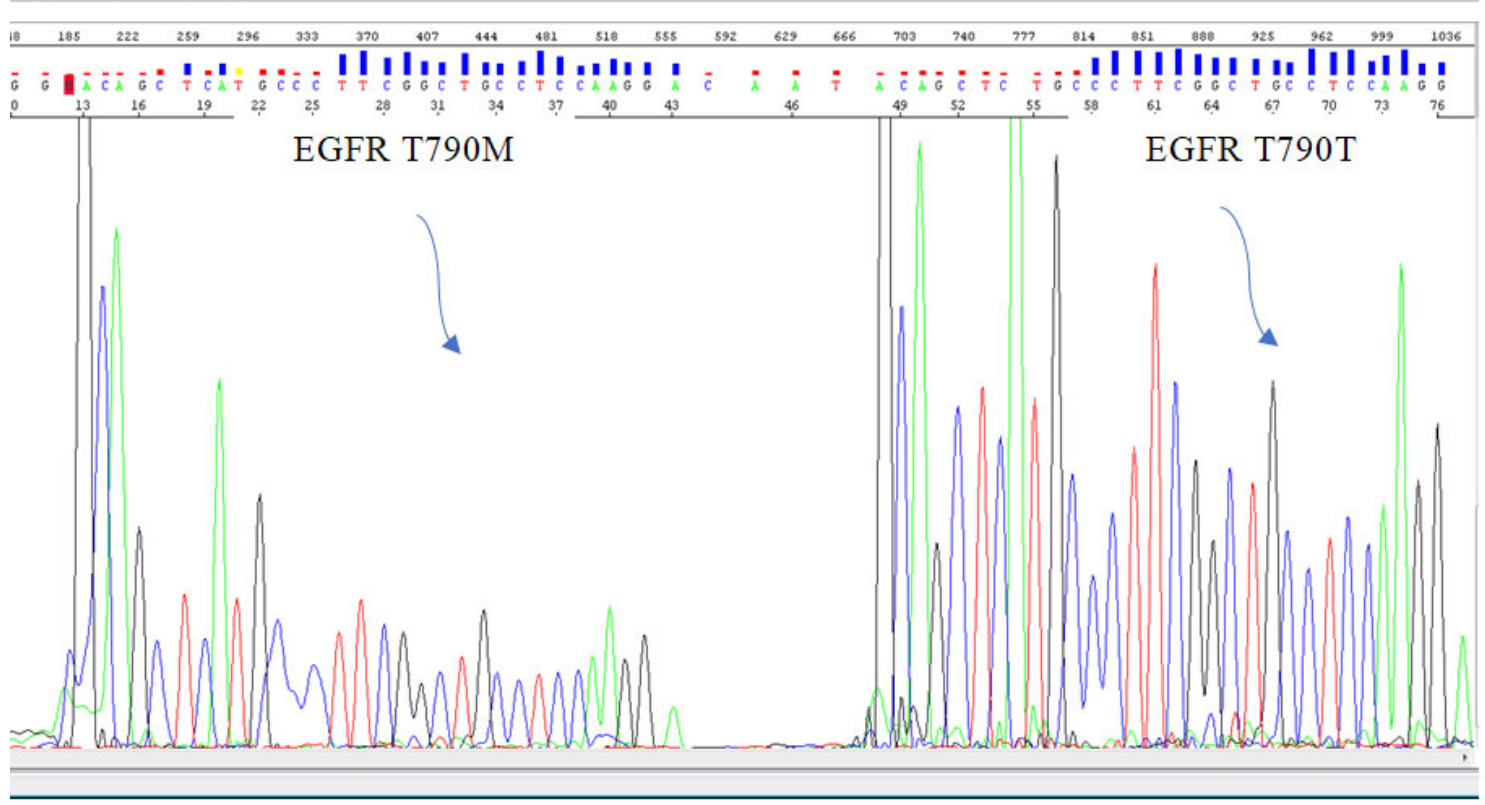

Figure 4. Electropherogram of a T790M positive FFPE sample

\subsubsection{Comparison detection of EGFR L858R, T790M us- ing FFPE samples}

Out of the forty-one samples that were tested for T790M, three were tested by pyrosequencing, all three were negative by both pyrosequencing and ASMS. Of the remaining thirty-eight samples that were compared with TruSeq, the seven positives and thirty-one negatives were all in $100 \%$ concordance with ASMS (see Table 5). Of the twenty samples tested by pyrosequencing for L858R, all but one sample were in concordance with ASMS, and of the forty-five samples tested by TruSeq for L858R, all the three positives and forty-two negative samples were in concordance with ASMS. Presently, the selection of targeted chemotherapy for late stage cancers has been based on molecular diagnostics platforms that were either developed for the detection of pathogens (e.g. Real time PCR) or for detecting germ line mutations (NGS). Clinical samples used for both real time PCR and NGS carry overwhelming copies of the intended molecular target, hence detection of intended molecular targets have been within the limits of respective technologies. However, detection of somatic mutations in early stages of cancer is becoming one of the main strategies for successful treatment of cancer, and this requires molecular technologies that could detect samples carrying low copy numbers. Further, incidence of somatic mutations in other cancer types such as ovarian, esophageal, etc. have not been reported, and may warrant re-evaluation of actionable somatic mutations. What is required for detection of mutations in early cancer stages is a molecular platform that could detect respective somatic mutations in samples carrying low copies of the mutant allele.

In addition to having both wild and the mutant allele in the same assay, ASMS technology could also accommodate more than one target, and hence becomes cost effective by testing multiple targets. Presently there are three DNA sequencing platforms, Sanger sequencing, pyrosequencing and next generation sequencing (e.g. TruSeq). In conventional Sanger sequencing/capillary electrophoresis, the florescent signal from a sample with low mutant copies is suppressed by the wild type signal such that the mutant signal may be indistinguishable from the noise, and thus only could be applied to samples with higher tumor content. ASMS technology separates the mutant signal from that of the wild type, hence the mutation can be detected with low copy numbers. NGS technologies have eleven enzyme mediated steps, such that cumulative errors could affect the accuracy. In order to minimize these errors NGS needs high copies of mutant in the sample. ASMS is an amplicon/sequencing technology and is determined by two primers in the PCR reaction and one sequencing primer, thus reducing the chance of error. In pyrosequencing, interpreting peak heights is subject to experienced technical expertise, and therefore it is subjective. ASMS generates an electropherogram specific to the mutation of intertest and can be analyzed by software, thus is not dependent on subjective interpretation. Further, we reported comparison of ASMS with other molecular technologies. ${ }^{[10]}$

In summary, the study shows that ASMS is in concordance with pyrosequencing and TruSeq in detecting actionable EGFR driver mutations from clinical samples. Further, 
ASMS can detect somatic mutation alleles at very low copy numbers. This data suggests that ASMS is suitable to detect actionable somatic mutations in early stages of cancer, and suitable to re-evaluate incidence of actionable somatic mutations in cancers where incidence of these mutations has not been reported.

\section{Conflicts OF InTEREST Disclosure}

Thuraiayah Vinayagamoorthy is President of MultiGEN Diagnostics, Inc.

\section{REFERENCES}

[1] Ladanyi M, Pao W. Lung adenocarcinoma: guiding EGFR-targeted therapy and beyond. Mod Pathol. 2008 May; 21 Suppl 2: S16-22. PMid:18437168. https://doi.org/10.1038/modpathol. 3801 018

[2] Pao W, Miller V, Zakowski M, et al. EGF receptor gene mutations are common in lung cancers from "never smokers" and are associated with sensitivity of tumors to gefitinib and erlotinib. Proc Natl Acad Sci U S A. 2004 Sep 7;101(36):13306-11. PMid:15329413. https://doi.org/10.1073/pnas.0405220101

[3] Kenneth S, Thress Roz Brant T. Hedley Carr cSimon Deardend Suzanne Jenkins e Helen Brownd Tracey Hammettf Mireille CantarinigJ. Carl Barretta. EGFR mutation detection in ctDNA from NSCLC patient plasma: A cross-platform comparison of leading technologies to support the clinical development of AZD9291. Lung Cancer. 2015 December; 90(3): 509-515. PMid:26494259. https://doi.org/10.1016/j.lungcan.2015.10.004

[4] Sandrine Dufort, Marie-Jeanne Richard, Sylvie Lantuejoul, et al. Pyrosequencing, a method approved to detect the two major EGFR mutations for anti EGFR therapy in NSCLC J Exp Clin Cancer Res. 2011; 30(1): 57. PMid:21575212. https://doi.org/10.1186/ 1756-9966-30-57

[5] Vinayagamoorthy T, Zhang D, Ye F, Vinayagamoorthy D, Hodkinson R. Can detection of Braf p.V600E mutation be improved? Compari- son of allele specific multiplex sequencing to present tests. Journal of Solid Tumors. 2017; 7(2): 14. https ://doi.org/10.5430/js t.v7n2p14

[6] Sandrine Dufort, Marie-Jeanne Richard, Sylvie Lantuejoul, et al. Pyrosequencing, a method approved to detect the two major EGFR mutations for anti EGFR therapy in NSCLC. J Exp Clin Cancer Res. 2011; 30(1): 57. https://doi.org/10.1056/NEJMoa1709684

[7] Gillian Ellison, Guanshan Zhu, Alexandros Moulis, et al. EGFR mutation testing in lung cancer: a review of available methods and their use for analysis of tumour tissue and cytology samples. J Clin Pathol. 2013; 66:79-89. PMid:23172555. https ://doi .org/10.1 136/jclinpath-2012-201194

[8] Martínez-Carretero C, Pascual FI, Rus A, et al. Detection of EGFR mutations in patients with non-small cell lung cancer by high resolution melting. Comparison with other methods Clin Chem Lab Med. 2017 Oct 26; 55(12): 1970-1978. PMid:28432840. https : //doi.org/10.1515/cclm-2016-0353

[9] Hee JK, Seo YO, Wan SK, et al. Clinical investigation of EGFR mutation detection by pyrosequencing in lung cancer patients. Oncol Lett. 2013 Jan; 5(1): 271-276. PMid:23255934. https : //doi .or g/10.3892/ol.2012.950

[10] Moorthy, Thurai. An algorithm to evaluate the efficacy of detecting somatic mutations. J Solid Tumors. 2018; 8(2): 25. https: //doi.org/10.5430/jst.v8n2p25 